\title{
Correlation of Flame Speed with Stretch in Turbulent Premixed Methane/Air Flames
}

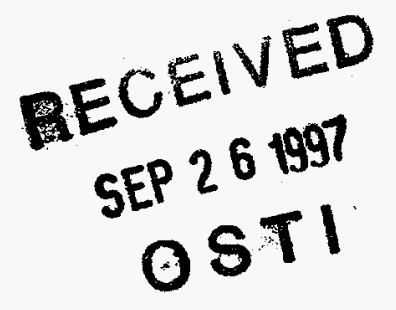

JACQUELINE H. CHEN and HONG G. IM

Combustion Research Facility, Mail Stop 9051

Sandia National Laboratories

Livermore, California 94551-0969

\section{Introduction}

\begin{abstract}
The submitted manusoripi hae been authored by a contrector of the Unired States Govermment under contract. Aooordingly the United Stater Govexnment retains a non-erchiusive, rotralty-free license to publish or $\mathrm{xe}$. produce the published form of this contribution, or allow others to do so, for United Stater Government puxposen:
\end{abstract}

In the flamelet approach of turbulent premixed combustion, the flames are modeled as a wrinkled surface whose propagation speed, termed the "displacement speed," is prescribed in terms of the local flow field and flame geometry [1]. Theoretical studies [2] suggest a linear relation between the flame speed and stretch for small values of stretch,

$$
S_{L} / S_{L}^{0}=1-M a K a,
$$

where $S_{L}^{0}$ is the laminar flame speed, $K a=\kappa \delta_{F} / S_{L}^{0}$ is the nondimensional stretch or the Karlovitz number, and $M a=\mathcal{L} / \delta_{F}$ is the Markstein number. The nominal flame thickness, $\delta_{F}$, is determined as the ratio of the mass diffusivity of the unburnt mixture to the laminar flame speed. Thus, the turbulent flame model relies on an accurate estimate of the Markstein number in specific flame configurations. Experimental measurement of flame speed and stretch in turbulent flames, however, is extremely difficult. As a result, measurement of flame speeds under strained flow fields has been made in simpler geometries $[3,4]$, in which the effect of flame curvature is often omitted.

In this study we present results of direct numerical simulations of unsteady turbulent flames with detailed methane/air chemistry, thereby providing an alternative method of obtaining flame structure and propagation statistics. The objective is to determine the correlation between the displacement speed and stretch over a broad range of Karlovitz numbers. The observed response of the displacement speed is then interpreted in terms of local tangential strain rate and curvature effects.

\section{Numerical Method}

The numerical scheme is based on the solution of the Navier-Stokes, species and energy equations for a compressible gas mixture with temperature dependent properties. The explicit finite difference algorithm uses a fourth-order low storage Runge-Kutta scheme for time advancement and an eighth-order explicit spatial differencing scheme [5]. The chemical mechanism is based on a detailed $\mathrm{C}_{1}$ mechanism by Warnatz [6] with 17 species and 68 reversible reactions. The species mass diffusion is determined by prescribing the Lewis numbers of each species [7]. The molecular viscosity of the mixture is temperature dependent, while the thermodynamic properties (enthalpy, specific heat) are temperature and composition dependent. The Prandtl number is taken to be 0.708 .

The computations are initialized with a one-dimensional steady laminar flame profile. A fuel-lean mixture (equivalence ratio of 0.7 ) of methane/air is preheated to $800 \mathrm{~K}$ in the reactant freestream. The profiles are obtained from a one-dimensional steady code PREMIX [8], and the solution is allowed to adjust to the simplified transport in a one-dimensional DNS.

The turbulence is prescribed by an initial two-dimensional turbulent kinetic energy spectrum function [9] which is superimposed on the laminar flame. The ratio of the turbulence intensity, $u^{\prime}$, to $S_{L}^{0}$ is taken to be 10 , and the ratio of the integral eddy scale, $L_{11}$, to $\delta_{F}$ is 2.77 . The turbulence Reynolds number based 


\section{DISCLAIMER}

This report was prepared as an account of work sponsored by an agency of the United States Government. Neither the United States Government nor any agency thereof, nor any of their employees, makes any warranty, express or implied, or assumes any legal liability or responsibility for the accuracy, completeness, or usefulness of any information, apparatus, product, or process disclosed, or represents that its use would not infringe privately owned rights. Reference herein to any specific commercial product, process, or service by trade name, trademark, manufacturer, or otherwise does not necessarily constitute or imply its endorsement, recommendation, or favoring by the United States Government or any agency thereof. The views and opinions of authors expressed herein do not necessarily state or reflect those of the United States Government or any agency thereof. 


\section{DESCLAMIER}

Portions of this docoment may be illegible in electronic image products. Imoges are produced from the best available origion coscomeror 
on $L_{11}$ and the unburned gas properties at $800 \mathrm{~K}$ is 181 . The computational domain size is $0.67 \mathrm{~cm}$, or $21.6 \delta_{F}$, in the directions parallel and perpendicular to the laminar flame. The domain is resolved into 750 uniform grid points in each direction.

\section{The Displacement Speed}

The direct numerical simulation results are used to evaluate the displacement speed of an isoline representing the flame front. The isoline chosen corresponds to the $10 \%$ value of the unburnt methane mass fraction which is approximately the location of maximum heat release in the flame. The density-weighted displacement speed of the flame relative to the local gas velocity is defined as:

$$
S_{d}^{*} \equiv \frac{\rho S_{d}}{\rho_{u}}=-\frac{\dot{\omega}_{\alpha}}{\rho_{u}|\nabla Y|}-\frac{\frac{\partial}{\partial \eta}\left(\rho D_{\alpha} \frac{\partial Y}{\partial \eta}\right)}{\rho_{u}|\nabla Y|}-\frac{\rho D_{\alpha}}{\rho_{u}}(\nabla \cdot \mathbf{n})
$$

where $\rho_{u}$ denotes the density in the unburned state of the mixture, and $\eta$ is the direction normal to the flame. The density-weighted formulation eliminates dilatational effects on the displacement speed, and yields a relatively constant value across the reaction zone [13]. Here, the subscript $\alpha$ is the index of the species for which the mass fraction isocontour is being tracked. The unit normal vector of the isoline is defined as

$$
\mathbf{n}=-\frac{\nabla Y_{\alpha}}{\left|\nabla Y_{\alpha}\right|}
$$

Equation (2) shows that the value of the displacement speed is a result of the balance between reaction (first term on RHS), normal diffusion (second term on RHS), and tangential diffusion (third term on RHS), and is modulated by the value of the scalar gradient at the location where it is measured. It is noted that the tangential diffusion term is linearly proportional to the local curvature $[10,12]$.

\section{Results and Discussion}

The correlation of the displacement speed with Karlovitz number is shown in Fig. 1. It is observed that there exist two distinct branches depending on the sign of the displacement speed. The upper branch shows that the displacement speed decreases with an increase in $K a$ (and therefore positive $M a$ from the definition in Eq. (1)), which is consistent with conventional description. In this branch, a linear curve fit for $K a$ less than unity gives a Markstein number of 1.55 , which is in reasonable agreement with the value 1.27 obtained from the counterflow premixed flame calculations under the same upstream conditions. This confirms that, for small Karlovitz numbers (less than 1), tangential strain rate has a stronger influence on the flame speed than curvature.

The negative branch in Fig. 1 may seem counter-intuitive, as it appears that for some portion of the flame the displacement speed increases with the Karlovitz number. However, it is found that this branch occurs in the region of large positive (convex to the upstream) curvature, where the center of curvature is located in the burnt gases. In this case, the flame is convectively pulled upstream by strong turbulent eddies, such that the magnitude of the diffusive flux tangential to the flame front exceeds that of the adverse convective flux [10]. As a result, the flame retreats back towards products and exhibits negative displacement speed. The positive slope in the negative branch in Fig. 1 thus simply shows that the higher the positive curvature becomes, the faster is the retreating flame speed.

The negative flame speed can also be achieved purely by excessive tangential straining, when normal diffusion exceeds reaction locally, again to counteract the adverse convective fluxes [11]. In the earlier stage of the DNS results, we observed the negative displacement speed for very large values of positive Karlovitz number, shown by the linear segment in Fig. 2 for $K a>1$.

As a general remark regarding the present turbulent premixed flame data, we observe that large negative values of Karlovitz numbers are found to be mainly due to the effect of strong curvatures; for these cases the correlation shows nonlinear behavior. For large negative curvatures (upper branch in Fig. 1), the 
displacement speed is enhanced due to increased radical and fuel consumption rates resulting from focusing of $\mathrm{H}$ atom at the cusp [13]. Local flame curvatures in excess of 2 thermal thicknesses are observed as a result of the intense turbulence. For radius of curvature less than 0.25 nominal flame thickness, the flame undergoes mutual annihilation with neighboring flames. The process of upstream flame-flame interaction then counteracts the propensity of the flame to propagate faster as a result of differential diffusion of $\mathrm{H}$ atom from behind the flame. The balance between these two processes determines the displacement speed for large negative curvatures. On the other hand, when the flame is positively curved, in the limit of large curvature, the displacement speed is linearly proportional to the local curvature, or diffusion tangential to the flame surface [10].

The statistical importance of the various branches in the correlation is shown in a probability density function ( $p d f)$ of the stretch rate shown in Fig. 3. The pdf has a positive mean value of 0.23 and a root mean square value of 1.49. Most of the area under the pdf is between a Karlovitz number of -2.5 and 1.0 , in a region where both strain and curvature effects are present. While the mean stretch rate is positive, a significant fraction of the flame is experiencing negative stretch at any given instant as a result of turbulence induced wrinkling of the flame.

\section{Acknowledgments}

This research was supported by the United States Department of Energy, Office of Basic Energy Sciences, Chemical Sciences Division. The authors thank.Dr. Tarek Echekki for many insightful discussions.

\section{References}

[1] Williams, F. A., "The Mathematics of Combustion" (J. D. Buckmaster, Ed.), Society for Industrial and Applied Mathematics, Philadelphia, 1985, pp. 97-131.

[2] Matalon, M. and Matkowsky, B. J., J. Fluid Mech., 124: 239 (1982).

[3] Taylor, S. C., Ph. D. Thesis, University of Leeds, Leeds, 1991.

[4] Tseng, L. K., Ismail, M. A., and Faeth, G. M., Combust. Flame, 95: 410 (1993).

[5] Kennedy, C. A. and Carpenter, M. H., Appl. Num. Math., 14: 3 (1996).

[6] Warnatz, J., Maas, U. and Dibble, R., Combustion: Physical and Chemical Foundations, Modelling, Pollutant Formation, Springer-Verlag, Heidelberg, Germany, 1996.

[7] Smooke, M. D. and Giovangigli, V., in Reduced kinetic mechanisms and asymptotic approximation for methane-air flames (Smooke, M. D. Ed.), Lecture Notes in Physics, no. 384, pp. 1-28, Springer-Verlag, New York, 1991.

[8] Kee, R. J., Grcar, J. F., Smooke, M. D. and Miller, J. A., Sandia National Laboratories Report SAND85-8240, 1985.

[9] Hinze, J. O., Turbulence, McGraw Hill, New York, 1975.

[10] Gran, I. R., Echekki, T. and Chen, J. H., Twenty-Sixth (Int.) Symposium on Combustion, The Combustion Institute, 1996, pp. 323-329.

[11] Libby, P. A. and Williams, F. A., Combust. Flame, 44: 287 (1982).

[12] Echekki, T. and Chen, J. H., The Contribution of Curvature to the Propagation of Turbulent Premixed Methane-Air Flames, in preparation, 1997.

[13] Echekki, T. and Chen, J. H., Combust. Flame, 106: 184 (1996). 


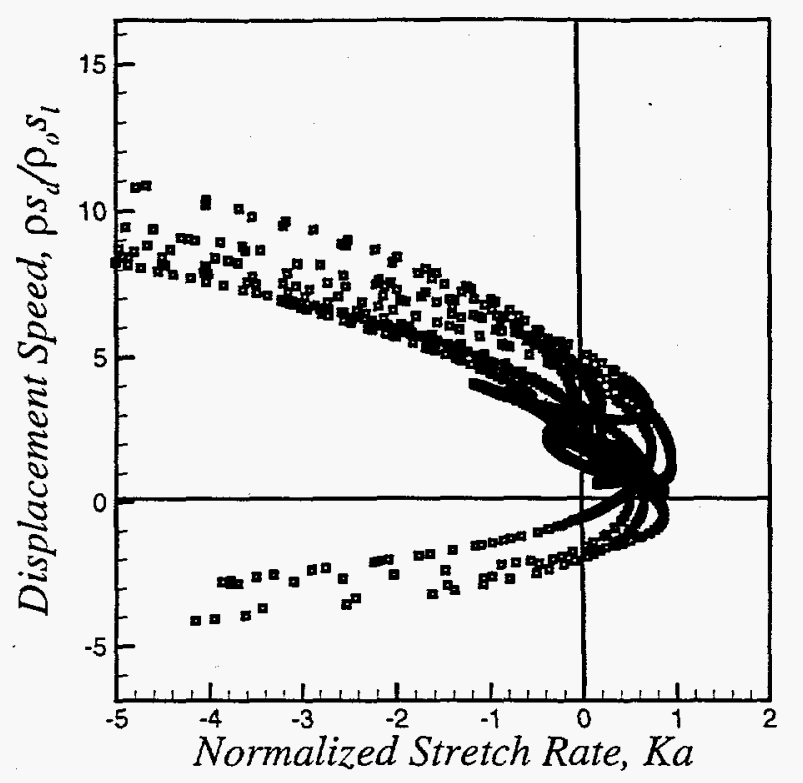

Figure 1: Correlation of displacement speed with stretch rate for methane/air flame at an equivalence ratio of 0.7 .

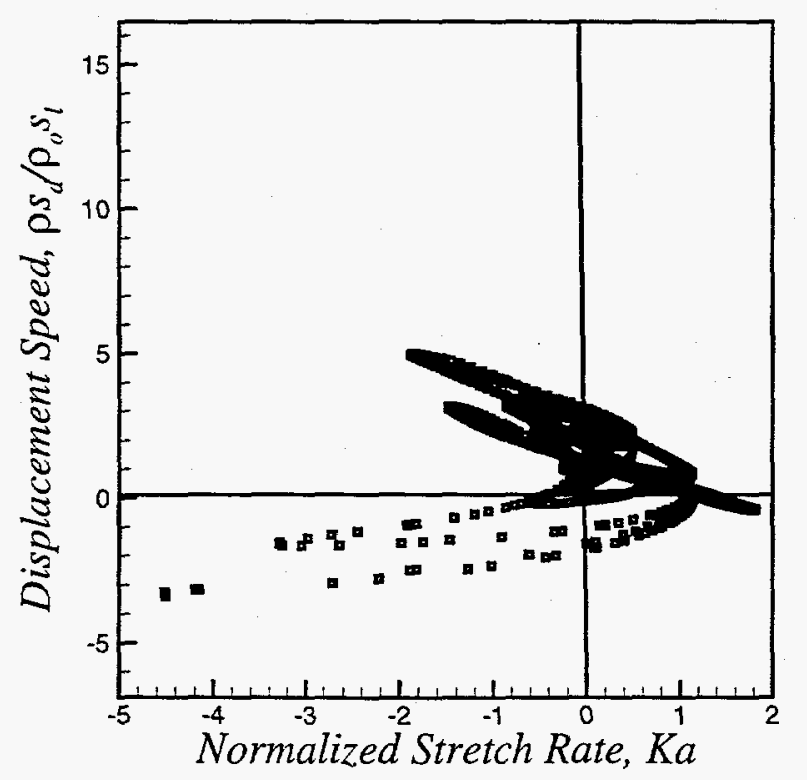

Figure 2: Correlation of displacement speed with stretch rate for methane/air flame at an equivalence ratio of 0.7 showing negative flame speed for $K a>1$.

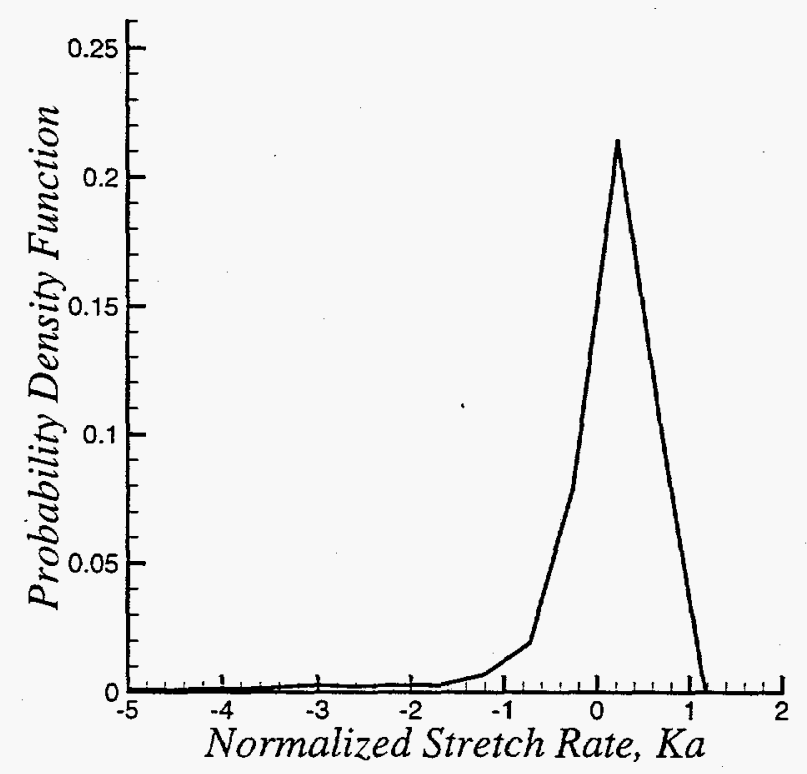

Figure 3: Probability density function of stretch rate at $10 \%$ unburnt $\mathrm{CH}_{4}$ 